\title{
Importance Sampling Simulation of Population Overflow in Two-node Tandem Networks
}

\author{
Victor F. Nicola Tatiana S. Zaburnenko \\ Faculty of Electrical Engineering, Mathematics and Computer Science \\ University of Twente, Enschede, The Netherlands \\ Email: \{v.f.nicola,t.s.zaburnenko\}@ewi.utwente.nl
}

\begin{abstract}
In this paper we consider the application of importance sampling in simulations of Markovian tandem networks in order to estimate the probability of rare events, such as network population overflow. We propose a heuristic methodology to obtain a good approximation to the 'optimal' state-dependent change of measure (importance sampling distribution). Extensive experimental results on 2-node tandem networks are very encouraging, yielding asymptotically efficient estimates (with bounded relative error) where no other state-independent importance sampling techniques are known to be efficient. The methodology avoids the costly optimization involved in other recently proposed approaches to approximate the 'optimal' statedependent change of measure. Moreover, the insight drawn from the heuristic promises its applicability to larger networks and more general topologies.
\end{abstract}

\section{Introduction}

Queueing network models are among the most natural for quantitative analysis, capacity planning and buffer dimensioning of logistics, manufacturing and communication systems and networks. Owing to their rarity, efficient simulation of buffer overflow events in queuing network models has been a challenging problem. Among the most effective methodologies researched and applied so far are those based on importance sampling (see, e.g., [8], [2], [15]) and importance splitting (see, e.g., [13], [23], [12]) techniques. (Importance sampling is the methodology adopted in this paper.) However, to date, the success of these techniques has been limited to simulations of single server queues and some very simple queueing networks, under restrictive assumptions regarding the underlying arrival and service pro- in the network or that of the total network population, given some initial network state (e.g., starting from an empty network). Until recently, only state-independent importance sampling heuristics were developed and considered for analysis. In these heuristics, the change of measure is 'static' and independent of the network state (the number of customers at network nodes).

A relatively simple (and well known) heuristic change of measure for simulations of population overflow in queueing networks is that proposed in [20] and further investigated in [10] and [11]. However, even for the simplest Jackson queueing network (e.g., 2-node tandem network), the effectiveness of this heuristic is limited to only some region of the (arrival and service) parameters space (see [14], [5]). (We use the term 'effectiveness' interchangeably with 'asymptotic efficiency,' see Section 2.2 for a precise definition.) Effective bandwidth methods have been used to develop heuristics for simulating overflow of an individual buffer in some specific class of networks, e.g., feed-forward [9] and in-tree [7] fluid flow networks. Recently [16] proposed a heuristic change of measure for simulating the overflow of an individual buffer in queueing networks of general topology and arbitrary routing (i.e., including feedback). Stateindependence is a common feature of the heuristics mentioned above. Also, none of them is provably effective for simulating population overflow in networks with an arbitrary and feasible set of parameters ([14], [16], [5]).

For the two-node tandem network, it is shown in [18] that a state-dependent change of measure can be effective where, provably, no effective state-independent change of measure exists ([14], [5]). Work in [6] uses adaptive optimization techniques based on the method of cross-entropy [21] to approximate the 'optimal' state-dependent change of measure. More recently, a similar adaptive approach based on stochastic approximation is introduced in [1]. These approaches appear to be the most promising for annlinntinn to Markovian (Jackson) networks of general $t$

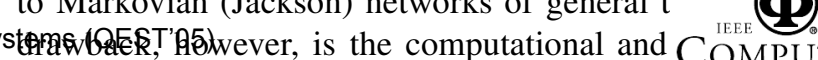


mands for large state-space models associated with large networks.

In this paper, we consider estimating the probability of population overflow in (Markovian) tandem queueing networks. As noted above, even for the simplest two-node tandem network, no state-independent change of measure exists which is effective for all feasible network parameters ([14], [18], [6], [16], [5]). The aim of the work presented in this paper is to use results and insights from these papers to develop a state-dependent change of measure which is sufficiently close to the 'optimal' without the need for a costly optimization procedure as in [6]. A key observation is that the 'optimal' change of measure depends on the network state only along and close to the boundaries (when one or more nodes are empty), and tends to become state-independent in the interior of the state-space. Therefore, if we can determine the change of measure along the boundaries and at the interior of the state-space, then we may be able to combine them appropriately to construct a state-dependent change of measure that approximates the 'optimal' one in the entire statespace. Preliminary experiments using the proposed methodology (dubbed 'state-dependent heuristic' or SDH in short) are very encouraging. Simulations to estimate the probability of population overflow in 2-node tandem networks produce asymptotically efficient estimates with bounded relative error.

In Section 2 we give some preliminaries and notation. We formally define the basic model and the probability of interest. A brief review of the importance sampling technique is also presented. In Section 3 we first introduce the basic idea and motivation for the proposed SDH. The approach is then applied to determine a heuristic change of measure for the 2-node tandem network. A time reversal argument is provided to justify this heuristic. Also, a modified heuristic leading to better efficiency is proposed. In Section 4 we present experimental results and comparisons with other known methods to estimate the probability of population overflow. Conclusions and a discussion of the advantages and challenges associated with the proposed methodology are given in Section 5.

\section{Preliminaries}

The queueing network model and associated notation are first introduced in Section 2.1. A brief review of importance sampling and definitions of some basic properties of simulation estimators are provided in Section 2.2.

\subsection{Model and notation}

Consider a Jackson network consisting of $n$ nodes (queues)

in tandem Customers arrive at the first node according to a $0-7695-2427-3 / 05$ Poisson process vith rate $\lambda$. The service time of a customer at node $i$ is exponentially distributed with rate $\mu_{i}, 1 \leqslant i \leqslant$ $n$. Customers that leave node $i$ join node $i+1$ (if $i<n$ ) or leave the network (if $i=n$ ). Each node has its own buffer, which is assumed to be infinite. We also assume that the queueing network is stable, i.e.,

$$
\lambda<\min _{i}\left\{\mu_{i}\right\} \text {. }
$$

Let $X_{i, t}(1 \leqslant i \leqslant n)$ denote the number of customers at node $i$ at time $t \geqslant 0$ (including those in service). Then the vector $\mathbf{X}_{t}=\left(X_{1, t}, X_{2, t}, \ldots, X_{n, t}\right)$ is a Markov process representing the state of the network at time $t$. Denote by $S_{t}$ the total number of customers in the network (network population) at time $t$, i.e., $S_{t}=\sum_{i=1}^{n} X_{i, t}$.

Assuming that the initial network state is $\mathbf{X}_{0}=(1,0, \ldots, 0)$ (i.e., upon a customer's arrival to an empty network), we are interested in the probability that the network population reaches some high level $L \in \mathbb{N}$ before returning to 0 . We denote this probability by $\gamma(L)$ and refer to it as the population overflow probability, starting from an empty network. It can be used to estimate other steady-state probabilities of interest. Consider, for example, the estimation of the steady-state probability that the network population exceeds a given level $L$. One way to estimate this probability is by observing a large number of 'true' regeneration cycles (e.g., with an empty network as a regeneration point). Then, an estimate of the steady-state probability of population overflow is the ratio of two (possibly independent) estimators [19]; one for the expected time at or above level $L$ in a cycle (the numerator), and the other for the expected cycle time (the denominator). The numerator could be estimated independently and more efficiently using the same importance sampling distribution as that used to estimate the population overflow probability introduced above (see Section 2.2).

\subsection{Importance sampling}

Importance sampling involves simulating the system (or model) under different underlying probability distributions so as to increase the frequency of typical sample paths leading to the rare event. Formally, denote by $\mathbb{P}$ and $\tilde{\mathbb{P}}$ the original and the new probability measures, respectively. Let $w$ be a sample path over the interval $[0, t]$. Then, the likelihood ratio associated with $w$ is given by $W_{t}(w)=\frac{P(w)}{\tilde{P}(w)}$, where $P(w)$ and $\tilde{P}(w)$ are the probabilities (or likelihoods) of sample path $w$ under the original and the new measure, respectively. An obvious condition is that $\tilde{P}(w)>0$ whenever $P(w)>0$. Starting from $\mathbf{X}_{0}$, define $\tau$ as the first time $S_{t}$ hits level $L$ or level 0 , then

$$
\gamma(L)=\mathbb{E} I_{\left\{S_{\tau}=L\right\}}=\tilde{\mathbb{E}} W_{\tau} I_{\left\{S_{\tau}=L\right.}
$$
where $U_{S T}, 65$ the likelihood ratio over the inter
and $\mathbb{E}$ are the expectations under the original : COMPUTER
SOCIETY 
change of measures, $\mathbb{P}$ and $\tilde{\mathbb{P}}$, respectively.

The variance of the estimator $\tilde{\mathbb{E}} W_{\tau} I_{\left\{S_{\tau}=L\right\}}$ is given by

$$
\tilde{\mathbb{E}} W_{\tau}^{2} I_{\left\{S_{\tau}=L\right\}}-(\gamma(L))^{2} .
$$

Thus, a variance reduction is obtained if $\tilde{\mathbb{P}}$ is chosen such that

$$
\tilde{\mathbb{E}} W_{\tau}^{2} I_{\left\{S_{\tau}=L\right\}}<\mathbb{E} I_{\left\{S_{\tau}=L\right\}} .
$$

The relative error is defined as the ratio of the standard deviation of the estimator over its expectation, i.e.,

$$
\sqrt{\frac{\tilde{\mathbb{E}} W_{\tau}^{2} I_{\left\{S_{\tau}=L\right\}}}{\gamma(L)^{2}}-1} .
$$

The estimator $\tilde{\mathbb{E}} W_{\tau} I_{\left\{S_{\tau}=L\right\}}$ is said to be asymptotically efficient if its relative error grows at sub-exponential (e.g., polynomial) rate as $L \rightarrow \infty$, (i.e., as $\gamma(L) \rightarrow 0$ ). This is a desirable property which may be achieved by a careful (and often complex) problem-specific analysis to determine the 'optimal' change of measure.

Formally, let $\lim _{L \rightarrow \infty} \frac{1}{L} \log \gamma(L)=\theta$. That is, $\theta$ is the asymptotic decay rate of the overflow probability $\gamma(L)$ as $L \rightarrow \infty$. Then, asymptotic efficiency is obtained if

$$
\lim _{L \rightarrow \infty} \frac{1}{L} \log \tilde{\mathbb{E}} W_{\tau}^{2} I_{\left\{S_{\tau}=L\right\}}=2 \theta .
$$

The estimator is said to have the bounded relative error property (which implies asymptotic efficiency) if its relative error is bounded in $L$ as $\gamma(L) \rightarrow 0$.

It is important to note that a change of measure may, in general, depend on the state of the system, even if the original underlying distributions do not depend on the system state. For instance, in a Markovian queueing network, the new arrival and service rates (to be used in importance sampling) may depend on the state of the network (i.e., the buffer content at each node). A state-dependent change of measure is more suited to affect the dynamics of the simulated system so as to follow the 'optimal' (most likely) path leading to the rare event. Indeed, a properly determined statedependent change of measure typically outperforms the 'optimal' state-independent one, if the latter exists (for theoretical and empirical evidence, see, e.g., [18], [4], [6]).

In the next section we propose an approach to approximate the 'optimal' state-dependent change of measure. If the approximation is sufficiently good, then asymptotic efficiency is achieved, as evidenced experimentally in Section 4.

\section{State-dependent heuristic (SDH)}

Recently proposed methods to determine state-dependent change of measures have some obvious drawbacks. It is not Proceedings of the Second International Conference Pn the Quantitative Evaluation 0-7695-2427-3/05 \$20.00 @ 2005 ltEEE to larger networks. Similarly, computational demands and large state-space limit the effectiveness of adaptive methods ([1], [6]). In this section we propose a new approach and use it to determine a state-dependent change of measure to estimate the probability of population overflow in 2 node tandem networks. No proofs of asymptotic efficiency are provided, however, the heuristics involved are motivated by arguments based on 'time-reversal' of large deviation paths [3] and are empirically shown to yield estimates with bounded (or linear in $L$ ) relative error. The general approach can also be applied to larger networks; preliminary experimental results for 3-node and 4-node tandem networks (not reported in this paper) are quite promising.

\subsection{Motivation}

The change of measure proposed in this section is inspired by theoretical and empirical results in [18] and [6]. These results indicate that the 'optimal' change of measure depends on the state of the network, i.e., the number of customers at the network buffers. Furthermore, this dependence is strong along the boundaries of the state-space (i.e., when one or more buffers are empty) and eventually (often quickly) disappears in the interior of the state-space (i.e., when the contents of all buffers in the network are sufficiently large). Capturing dependencies along the boundaries has shown to be very crucial for the asymptotic efficiency (or 'optimality') of the change of measure.

The above observation suggests that if we know the 'optimal' change of measure along the boundaries and in the interior of the state-space, then we might be able to 'construct' a change of measure that approximates the 'optimal' one over the entire state-space. If the approximation is sufficiently good, then the change of measure may yield asymptotically efficient estimators. Indeed, this is confirmed by empirical results in Section 4.1. To realize this idea we use heuristics based on combining known large deviations results and 'time-reversal' arguments, as explained in the following sections for the 2-node tandem network.

\subsection{SDH for the two-node tandem network}

The state of the 2-node network is completely determined by the continuous-time Markov chain (CTMC) process $\mathbf{X}_{t}=\left(X_{1, t}, X_{2, t}\right)$. Without loss of generality, we assume that $\lambda+\mu_{1}+\mu_{2}=1$. The change of measure to be used in importance sampling is also a CTMC (on the same statespace), however, with different arrival and service rates: $\tilde{\lambda}, \tilde{\mu_{1}}, \tilde{\mu_{2}}$, which, in general, may depend on the state of the network. In other words, let $\mathbf{x}=\left(x_{1}, x_{2}\right)$ be the state of the network at some time $t$. Then the new nuind nnd service rates at time $t$ are allowed to depend or

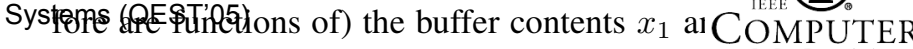
SOCIETY 
that the new change of measure does not necessarily correspond to a stable network, and, therefore, the inequality $\tilde{\lambda}<\min \left(\tilde{\mu_{1}}, \tilde{\mu_{2}}\right)$ need not to hold. As for the original network, we assume that $\tilde{\lambda}+\tilde{\mu_{1}}+\tilde{\mu_{2}}=1$.

Proposition 1 Define $[a]^{+}=\max (a, 0)$ and $[a]^{1}=$ $\min (a, 1)$, and let $0 \leq b \leq L$ be a fixed integer. The following equations describe the proposed change of measure for the 2-node tandem network:

$$
\begin{gathered}
\tilde{\lambda}\left(x_{2}\right)=\left[\frac{b-x_{2}}{b}\right]^{+} \mu_{1}+\left[\frac{x_{2}}{b}\right]^{1} \mu_{2} \\
\tilde{\mu_{1}}\left(x_{2}\right)=\left[\frac{b-x_{2}}{b}\right]^{+} \lambda+\left[\frac{x_{2}}{b}\right]^{1} \mu_{1} \\
\tilde{\mu_{2}}\left(x_{2}\right)=\left[\frac{b-x_{2}}{b}\right]^{+} \mu_{2}+\left[\frac{x_{2}}{b}\right]^{1} \lambda .
\end{gathered}
$$

Note that the new arrival and service rates depend on the state of the network only through $x_{2}$, the buffer content at the second node. The above heuristic (SDH) suggests two change of measures $\left(\mathcal{M}_{1}\right)$ and $\left(\mathcal{M}_{2}\right)$ (as indicated schematically in Figure 3).

Along the boundary, $x_{2}=0$, the change of measure $\left(\mathcal{M}_{1}\right)$ is given by:

$$
\tilde{\lambda}=\mu_{1}, \tilde{\mu_{1}}=\lambda, \tilde{\mu_{2}}=\mu_{2} .
$$

When $x_{2} \geq b$, the change of measure $\left(\mathcal{M}_{2}\right)$ is given by:

$$
\tilde{\lambda}=\mu_{2}, \tilde{\mu_{1}}=\mu_{1}, \tilde{\mu_{2}}=\lambda .
$$

In the interim, $0<x_{2}<b$, the new rates are simply linear interpolation from their values at $x_{2}=0$ to their values at $x_{2}=b$.

Let us follow a sample path starting from an arrival to an empty network, the proposed change of measure implies the following: Initially, and while $x_{2}=0$, exchange the arrival rate $(\lambda)$ with the service rate at node $1\left(\mu_{1}\right)$, i.e., start with the first node being unstable and the second node is stable. As the buffer content in the second buffer increases in the range $\left(0<x_{2}<b\right)$, gradually and simultaneously reduce the 'load' on the first node while increasing the 'load' on the second node. When the buffer content at the second node reaches (and exceeds) level $b$, exchange the arrival rate $(\lambda)$ with the service rate at node $2\left(\mu_{2}\right)$. That is, in the interior (and as long as $x_{2} \geq b$ ) the second node is unstable, the first node is stable (resp. unstable) if $\mu_{2}<\mu_{1}$ (resp. if $\mu_{1}<\mu_{2}$ ), and the new rates do not depend on the network state.

Remark 1 Note that $b$ is the range (number) of boundary Proceedings of the Sels for which the change of measuresdepends on the net-

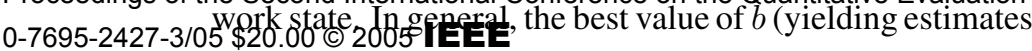

with lowest variance) may depend on the set of network parameters as well as the overflow level $L$. Empirical results in Section 4 indicate that, for some regions in the parameter space, the best $b$ is quite robust and does not change with the level $L$. For other regions, the best $b$ may vary slightly from one parameter point to another, and may also depend on $L$. It is also important to note that for $\mu_{1}<\mu_{2}$ (resp. $\mu_{2}<\mu_{1}$ ), the above change of measure reduces to that in [20] when $b=\infty($ resp. $b=0)$.

\subsection{Time reversal argument}

The above change of measure can indeed be corroborated using 'time-reversal' [17] as argued in this section. The reverse time process of the 2-node tandem network being considered is also a 2-node tandem network in which the arrival rate is $\lambda$ and the service rate at Node 1 (resp. Node 2) is $\mu_{2}$ (resp. $\mu_{1}$ ). Unlike the forward time process, arrivals in the reverse time process enter the network at Node 2 and exit from Node 1 (see Figure 1).

Roughly speaking, according to [3], in the limit as $L \rightarrow \infty$, the most likely path to the rare set (e.g., population overflow) in the forward time process is the same path by which the reverse time process evolves, given that the latter starts from the rare set. Clearly, we need to know more precisely where the rare set is most likely to be hit. This, of course, depends on the service rates. According to [3], if $\mu_{1}<\mu_{2}$, then (asymptotically) this is the state $\left(x_{1}=L, x_{2}=0\right)$. Alternatively, if $\mu_{2}<\mu_{1}$, then (asymptotically) this state is $\left(x_{1}=0, x_{2}=L\right)$.

It is important to note, however, that for large but finite $L$, the hitting state at the rare set is no longer at one of the extremes $(L, 0)$ or $(0, L)$ but somewhere along the line $x_{1}+$ $x_{2}=L$. That is, both Node 1 and Node 2 may be nonempty (and possibly both are unstable) upon entry into the rare set at some state $\left(L_{1}, L_{2}\right)$ such that $L_{1}+L_{2}=L$. This is a key to the justification of the proposed change of measure via time reversal argument as explained below.

Let $\mu_{1}<\mu_{2}$, then the reverse time process starts at $\left(L_{1}, L_{2}\right)$, with $L_{1} \gg L_{2}$. Node 2 has external arrival rate $\lambda$ and initially its departure rate is $\mu_{1}$ (not $\mu_{2}$ !), thus it empties at rate $\mu_{1}-\lambda$. In the meantime, Node 1 has input and departure rates equal to $\mu_{1}$, thus it is initially 'critical' (i.e., no build up). Node 2 empties first, and when it does, its arrival and departure rates are equal to $\lambda$. At that time, Node 1 has arrival rate $\lambda$ and departure rate $\mu_{1}$, thus it empties at rate $\mu_{1}-\lambda$.

Note that departures (resp. arrivals) in reverse time correspond to arrivals (resp. departures) in forward time. It follows that along the most likely path to population overflow, Node 1 is initially unstable (arrival rate $\mu_{1}$ and cervice rate $\lambda)$ and thus builds up at rate $\mu_{1}-\lambda$, while Noc

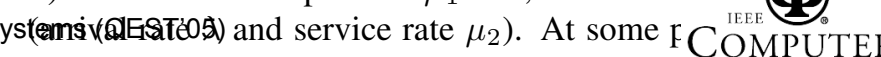
SOCIETY 
hitting the rare set, Node 1 becomes 'critical' and the number of customers stops building up (corresponding to arrival and service rates equal to $\mu_{1}$ ). At the same time, Node 2 starts to build up at rate $\mu_{1}-\lambda$ (corresponding to arrival rate $\mu_{1}$ and service rate $\lambda$ ).

A similar time reversal argument can be pursued when $\mu_{2}<$ $\mu_{1}$. In this case, the reverse time process starts at $\left(L_{1}, L_{2}\right)$, with $L_{2} \gg L_{1}$. Node 2 is backlogged, its departure rate is $\mu_{2}$ and its arrival rate is $\lambda$, thus it empties at rate $\mu_{2}-$ $\lambda$. In the meantime, Node 1 is 'critical,' having input and departure rates equal to $\mu_{2}$ (since $\mu_{2}<\mu_{1}$ ), thus no build up. When Node 2 empties, its arrival and departure rates are equal to $\lambda$. At that time, Node 1 has arrival rate $\lambda$ and service rate $\mu_{1}$, thus it empties at rate $\mu_{1}-\lambda$.

It follows that along the most likely path to population overflow, Node 1 is initially unstable (arrival rate $\mu_{1}$ and service rate $\lambda$ ), and Node 2 is initially stable (input rate $\lambda$ and service rate $\mu_{2}$ ). Soon after, Node 1 turns stable with arrival and departure rates equal to $\mu_{2}$ (since $\mu_{2}<\mu_{1}$ ). At the same time, Node 2 starts to build up at rate $\mu_{2}-\lambda$ (corresponding to input rate $\mu_{2}$ and service rate $\lambda$ ).

It is now clear that in both cases $\left(\mu_{1}<\mu_{2}\right.$ and $\left.\mu_{2}<\mu_{1}\right)$, the forward time process will (qualitatively) follow the same most likely path to population overflow (see Figure 2): initially Node 1 is unstable (arrival rate $\mu_{1}$ and service rate $\lambda$ ) and Node 2 is stable (arrival rate $\lambda$ and service rate $\mu_{2}$ ). After some time, Node 2 becomes unstable (input rate $\mu_{1}$ and departure rate $\lambda$ ) and Node 1 remains unstable (if $\mu_{1}<\mu_{2}$ ) or becomes stable (if $\mu_{2}<\mu_{1}$ ). Indeed, the heuristic proposed in Section 3.2 follows the above most likely (forward time) path scenario: initially $\left(x_{2}=0\right)$, the change of measure $\mathcal{M}_{1}$ is to exchange $\lambda$ and $\mu_{1}$. At some time, if and when $x_{2} \geq b$, the change of measure $\mathcal{M}_{2}$ is to exchange $\lambda$ and $\mu_{2}$. In the interim $\left(0<x_{2}<b\right)$, the change of measure is a linear interpolation of $\mathcal{M}_{1}$ and $\mathcal{M}_{2}$.

\subsection{Improved heuristic (SDH-A)}

It is important to note that the most likely path to the rare set (as predicted from time reversal) may not necessarily correspond to the actual (or 'optimal') one, particularly along the boundaries. Indeed, it turns out (as empirically observed) that the proposed change of measure described in Section 3.2 tends to 'over-bias' unstable nodes along and close to the boundaries (i.e., $x_{1}$ and/or $x_{2}$ close to 0 ). This observation prompted a modification of the heuristic in Proposition 1. The modified change of measure is described in the following proposition, and in this paper, we refer to it as $\mathrm{SDH}-\mathrm{A}$.

Proposition 2 Define $[a]^{+}=\max (a, 0)$ and $[a]^{1}=$ $\min (a, 1)$ and let $0<b<L$ be a fixed integer. The followProceedings of the Second Internationalcconference on the Quantitative Evaluation $0-7695-2427-3 / 05 \$ 20.00$ @ 2005 IEE 2-node tandem network:

$$
\begin{gathered}
\tilde{\lambda}\left(x_{2}\right)=\min \left(\mu_{1}, \mu_{2}\right) \\
\tilde{\mu_{1}}\left(x_{2}\right)=\left[\frac{b-x_{2}}{b}\right]^{+} \lambda+\left[\frac{x_{2}}{b}\right]^{1} \max \left(\mu_{1}, \mu_{2}\right) \\
\tilde{\mu_{2}}\left(x_{2}\right)=\left[\frac{b-x_{2}}{b}\right]^{+} \max \left(\mu_{1}, \mu_{2}\right)+\left[\frac{x_{2}}{b}\right]^{1} \lambda .
\end{gathered}
$$

Note that, unlike change of measure in Proposition 1, the new arrival rate is equal to $\min \left(\mu_{1}, \mu_{2}\right)$, independent of $x_{2}$. The modified heuristic (SDH-A) suggests two change of measures $\left(\mathcal{M}_{1}\right)$ and $\left(\mathcal{M}_{2}\right)$ (as indicated schematically in Figure 4).

Along the boundary, $x_{2}=0$, the change of measure $\left(\mathcal{M}_{1}\right)$ is given by:

$$
\tilde{\lambda}=\min \left(\mu_{1}, \mu_{2}\right), \tilde{\mu_{1}}=\lambda, \tilde{\mu_{2}}=\max \left(\mu_{1}, \mu_{2}\right) .
$$

When $x_{2} \geq b$, the change of measure $\left(\mathcal{M}_{2}\right)$ is given by:

$$
\tilde{\lambda}=\min \left(\mu_{1}, \mu_{2}\right), \tilde{\mu_{1}}=\max \left(\mu_{1}, \mu_{2}\right), \tilde{\mu_{2}}=\lambda .
$$

Let us follow a sample path starting from an arrival to an empty network, the proposed change of measure implies the following: The arrival rate is fixed at the minimum service rate. Initially, and while $x_{2}=0$, Node 1 is unstable with service rate $\lambda$, and Node 2 is stable with service rate $\mu_{2}$ (resp. $\mu_{1}$ ) if $\mu_{2}>\mu_{1}$ (resp. if $\mu_{1}>\mu_{2}$ ). When the buffer content at Node 2 reaches (and exceeds) level $b$, Node 2 is unstable with service rate $\lambda$, and Node 1 is stable or 'critical' (if $\mu_{1}=\mu_{2}$ ).

For the 2-node tandem network, the above modified heuristic (SDH-A) performs comparably or slightly better than SDH (see experimental results in Section 4.1).

\section{Experimental results}

Importance sampling to estimate the probability of population overflow $(\gamma(L))$ involves generating, say, $N$, independent and identically distributed (i.i.d.) busy cycles (i.e., starting with an empty network). In each cycle, the change of measure described above is applied until either the population overflow event is reached or the network population returns to 0 .

Starting a cycle at time 0 , define $\tau_{L}$ as the instant when the network population reaches level $L$ for the first time. Similarly, define $\tau_{0}$ as the instant when the networl nam. lation returns to 0 for the first time. The indica Systemps ${ }_{L}\left(\mathrm{QEST}^{\prime} O 5\right)$ takes the value 1 if the populati COMPUTER 
(level $L$ ) is reached in cycle $i$, otherwise it takes the value 0 . An unbiased estimator $\tilde{\gamma}$ of $\gamma(L)$ is given by

$$
\tilde{\gamma}=\frac{1}{N} \sum_{i=1}^{i=N} I_{i} W_{i},
$$

where $W_{i}$ is the likelihood ratio associated with cycle $i$. The second moment of the random variable $I W$ is estimated by

$$
\tilde{\gamma^{2}}=\frac{1}{N-1} \sum_{i=1}^{i=N} I_{i} W_{i}^{2} .
$$

The variance $\operatorname{VAR}(\tilde{\gamma})$ and the relative error $R E(\tilde{\gamma})$ of the importance sampling estimator $\tilde{\gamma}$ follow from:

$$
\begin{gathered}
V A R(\tilde{\gamma})=\frac{1}{N}\left(\tilde{\gamma}^{2}-(\tilde{\gamma})^{2}\right), \\
R E(\tilde{\gamma})=\frac{\sqrt{\operatorname{VAR(\tilde {\gamma })}}}{\tilde{\gamma}} .
\end{gathered}
$$

Another useful measure for comparing the efficiency of different estimators (e.g., different importance sampling heuristics) is the 'relative time variance' $(R T V)$ product, which is defined as the simulation time (in seconds) multiplied by the squared relative error of the estimator. Equivalent measures of efficiency are used in the literature, but may be termed differently. As the estimate becomes more stable, its $R T V$ tends to a constant value, which is smaller for a more efficient estimator. Therefore, $R T V$ can be used as an indicator to test convergence, to control accuracy, and to compare the efficiency of different estimators. For example, if $R T V_{2}$ (for Estimator 2) is larger than $R T V_{1}$ (for Estimator 1), then it will take Estimator 2 a longer simulation time to reach the same accuracy. The ratio $R T V_{2} / R T V_{1}$ represents the efficiency gain when using Estimator 1 relative to that when using Estimator 2. As in some literature, we refer to it as the variance reduction ratio $(V R R)$ and use it for comparing the efficiency of different importance sampling heuristics in the next section.

\subsection{Simulations of the two-node tandem network}

In this section we consider the 2-node tandem network and present experimental results obtained by using the proposed state-dependent change of measures (SDH and SDH-A), described in Section 3.2 and Section 3.4, respectively. Comparisons with other state-dependent heuristics (determined adaptively) are also presented.

It is shown empirically and/or proven formally ([14], [5]) that the well-known state-independent heuristic in [20] (here referred to as PW) yields asymptotically efficient estimates with bounded relative error only in some regions of

Proceedings of the Secondilnternational cronferences of the 0-7695-2427-3/05 \$20.00 @ 2005 IEEE corresponds to a stable network). Based on empirical results in [5], the feasible parameter space is divided into the following 3 regions, depending on the observed asymptotic properties of the PW estimator (see Figure 5):

Region I (BRE)- PW yields estimates with bounded relative error; Region II (ERE)- PW yields estimates with exponentially growing relative error; and Region III (IRE)- PW yields estimates with infinite variance/relative error.

Results obtained using the PW heuristic are reported to show where it does (or doesn't) work and how it compares with SDH and other heuristics when it works well. Comparisons with the state-dependent change of measure determined using the adaptive methodology in [6] (here referred to as SDA) are also included. As in SDH, SDA aims to approximate the 'optimal' state-dependent change of measure; however, the 'optimal' transition probabilities (at each state) are determined adaptively during simulation. SDA also assumes state-dependence over a (small) number $(b)$ of boundary layers. In either SDH or SDA, the best $b$ can be determined by repeating the simulation for increasing $b$, possibly starting with $b=0$ (i.e., no state-dependence). The best $b$ is the one that yield the maximum efficiency (i.e., when $R T V$ reaches a stable minimum). To approach a fair comparison, it is assumed in all experiments that the best $b$ (corresponding to each of the used methods) is pre-determined and is used to run the simulation. To verify the correctness of the simulation estimates, numerical results (using the algorithm outlined in [12]) are also included in the comparison tables. The experiments in this section are designed to demonstrate that the proposed state-dependent change of measures (described in Sections 3.2 and 3.4) always yield asymptotically efficient estimates (mostly with bounded relative error), also in those regions where no state-independent change of measure is known to be asymptotically efficient (i.e., Regions II and III in Figure 5). Therefore, 8 different experiments are performed, corresponding to 8 parameter points covering all regions in Figure 5. That is, 2 points in each of the 3 regions (BRE, ERE, and IRE) and 2 points along the line $\mu_{1}=\mu_{2}$. These points are representative and the results are consistent with extensive simulations (not included here) covering each and all regions in Figure 5.

In all simulation experiments, the same number of replications, namely, $10^{6}$, is used to obtain estimates of the population overflow probability $\gamma(L)$. These estimates are presented in Table 1 through Table 8; one table for each of the points indicated in Figure 5. For each estimate in the tables, we include the relative error (in percentage) as an indication of convergence and asymptotic efficiency. It is important to note, however, that the relative error displayed in the tables is not a good measure for comparisons, since the associated computational effort may be significantlv different from one method to another. In other words, a : Systimesequestiogy not necessarily mean a higher ef! 
the purpose of comparing the proposed heuristics (SDH and SDH-A) with that determined adaptively (SDA), we include $V R R$ (relative to $R T V$ of SDA) as a more objective measure of efficiency gains. Hence, $V R R=1$ for SDA, and a $V R R>1$ implies efficiency gain over SDA. It should also be noted that the experimental results presented here are obtained using basic and non-optimized implementations of SDH and SDA. Therefore, comparisons should not be interpreted as definitive, and may change in other experimental settings using different implementations. (A more optimized implementation of SDA is used in [6].)

A careful inspection of Table 1 through Table 8 leads us to make the following observations and comparisons between the tested heuristics, the state-independent (PW) and the state-dependent (SDA, SDH, and SDH-A):

BRE Region I (Tables 1 and 2):

All heuristics yield very accurate estimates with bounded relative error. Note, however, that for Point I-1, SDH and SDH-A have an 'optimal' $b$ equal to 0 (as found experimentally). For this point, SDA outperforms the other heuristics $(V R R<1)$. For Point I-2, SDH and SDH-A have an 'optimal' $b=\infty$ and yield efficiency gains over SDA $(1<V R R<3)$. As noted in Remark 1 , the heuristics SDH and SDH-A reduce to PW for $b=0$ (if $\mu_{2}<\mu_{1}$ ) and for $b=\infty$ (if $\mu_{1}<\mu_{2}$ ). The different results in Tables 1 and 2 are due to a slightly improved importance sampling implementation of SDH and SDH-A, in which the transition ending a busy cycle in the 'empty network' (rather than 'population overflow') state is not allowed.

ERE Region II (Tables 3 and 4):

Except for PW, all heuristics yield stable estimates. (The (*) next to $\mathrm{PW}$ in the tables is to indicate that its estimates are not stable.) For Point II-1, SDA, SDH, and SDH-A give bounded relative error, and SDA outperforms SDH and SDH-A $(.01<V R R<.07)$. (It is not clear why SDA gives much lower relative error for this point than it does for any other point). For Point II-2, the relative error seem to grow slowly and linearly with $L$, and SDH and SDH-A yield efficiency gains over SDA $(3<V R R<7)$.

IRE Region III (Tables 5 and 6):

Except for PW, all heuristics yield stable estimates. For Point III-1, SDA, SDH, and SDH-A give bounded relative error, with SDH and SDH-A slightly more efficient than SDA $(.2<V R R<3.5)$. For Point III-2, the relative error seem to grow linearly with $L$, and SDH and SDH-A yield efficiency gains over SDA $(4<V R R<6)$.

ERE/IRE Line $\mu_{1}=\mu_{2}$ (Tables 7 and 8):

Note that for Points IV-1 and IV-2, SDH and SDH-A are the same. Except for PW, all heuristics yield stable estimates with bounded relative error. SDH and SDH-A yield efficiency gains over SDA $(1<V R R<10)$. that the proposed heuristics SDH and SDH-A yield estimates with bounded relative error for points with $\mu_{2} \leq \mu_{1}$ (i.e., points in the lower triangle, below and including the line $\mu_{1}=\mu_{2}$ in Figure 5) as well as for points in the upper BRE Region I. Only for points in the upper ERE/IRE Regions II and III (with $\mu_{1}<\mu_{2}$ ), the SDH and SDH-A estimates have a linearly bounded relative error (see Tables 4 and 6). Note, however, that according to the interchangeability argument in [24], the probability of population overflow is invariant with respect to the placement order of nodes in a Jackson tandem network. Therefore, by interchanging the service rates $\left(\mu_{1}\right.$ and $\left.\mu_{2}\right)$, the overflow probability for points in the upper ERE/IRE Regions II and III can also be estimated with bounded relative error!

It should be noted that the above empirical results are based on simulation of the (simple) 2-node tandem network. Therefore, the efficiency comparisons are tilted in favour of SDA, which tends to be less effective for large state-space. More significant efficiency gains of SDH/SDH-A over SDA may be obtained for larger networks. Moreover, preliminary results (not reported here) from simulations of 3-node and 4-node tandem networks, with change of measures developed using the same approach, are quite encouraging.

\section{Conclusions and further work}

In this paper we have introduced a new approach to approximate the 'optimal' state-dependent change of measure to be used in importance sampling simulation of queueing networks. The approach is based on the observation that the 'optimal' (asymptotically efficient) change of measure depends on the state of the network most crucially along and close to the boundaries of the state-space. Therefore, if we can determine the 'optimal' change of measure along the boundaries and in the interior of the state space, then we may be able to construct a 'good' approximation over the entire state-space. The approach (which we refer to as SDH) is applied to derive a state-dependent change of measure for the estimation of population overflow in the 2-node tandem network. Empirical results show that importance sampling using this change of measure yields asymptotically efficient estimates (with bounded relative error), also where no stateindependent change of measure is known to be effective. Moreover, preliminary results (not reported here) from simulation of larger tandem networks using the same approach look promising. Developing similar heuristics for Jackson networks is also underway.

\section{Acknowledgment}

ystems wisesto thank Pieter-Tjerk de Boer for us sions relating to Figure 5. 


\section{References}

[1] T.P.I. Ahamed, V.S. Borkar and S.K. Juneja (2004). Adaptive importance sampling technique for Markov chains using stochastic approximation. Submitted manuscript.

[2] S. Asmussen and R.Y. Rubinstein (1995). Steady state rare events simulation in queueing models and its complexity properties. In Advances in Queueing: Theory, Methods and Open problems. J.H. Dshalalow (ed.), CRC Press, New York, 429-461.

[3] V. Anantharam, P. Heidelberger and P. Tsoucas (1990). Analysis of rare events in continuous time Markov chains via time reversal and fluid approximation. IBM Research Report RC 16280, Yorktown Heights, New York.

[4] P.T. de Boer (2000). Analysis and efficient simulation of queueing models of telecommunication systems. $\mathrm{PhD}$ Thesis, University of Twente.

[5] P.T. de Boer (2004). Analysis of sate-independent IS measures for the two-node tandem queue. International Workshop on Rare Event Simulation (RESIM'04), Budapest, Hungary.

[6] P.T. de Boer and V.F. Nicola (2002). Adaptive statedependent importance sampling simulation of Markovian queueing networks. European Transactions on Telecommunications 13 (4) 303-315.

[7] C.S. Chang, P. Heidelberger, S. Juneja and P. Shahabuddin (1994). Effective bandwidth and fast simulation of ATM in-tree networks. Performance Evaluation 20 45-65.

[8] M. Cottrell, J.-C. Fort and G. Malgouyres (1983). Large deviations and rare events in the study of stochastic algorithms. IEEE Trans. on Automatic Control 28 (9) 907-920.

[9] G. De Veciana, C. Courcoubetis and J. Walrand (1994). Decoupling bandwidths for networks: A decomposition approach to resource management for networks. In Proceedings of INFOCOM'94, IEEE Press, 466-473.

[10] M.R. Frater and B.D.O. Anderson (1989). Fast estimation of the statistics of excessive backlogs in tandem networks of queues. Australian Telecommun. Res. 23 (1) 49-55.

[11] M.R. Frater, T.M. Lenon and B.D.O. Anderson (1991). Optimally efficient estimation of the statistics of rare
[12] M.J.J. Garvels (2000). The splitting method in rare event simulation. PhD Thesis, University of Twente.

[13] P. Glasserman, P. Heidelberger, P. Shahabuddin and T. Zajic (1996). Multilevel splitting for estimating rare event probabilities. Operations Research 47 (4) 585600 .

[14] P. Glasserman and S-G. Kou (1995). Analysis of an importance sampling estimator for tandem queues. ACM Transactions of Modeling and Computer Simulation 5 (1) 22-42.

[15] P. Heidelberger (1995). Fast simulation of rare events in queueing and reliability models. ACM Transactions of Modeling and Computer Simulation 5 (1) 43-85.

[16] S.K. Juneja and V.F. Nicola (2003). Efficient simulation of buffer overflow probabilities in Jackson networks with feedback. ACM Transactions of Modeling and Computer Simulation. Under revision.

[17] F.P. Kelly (1979). Reversibility and Stochastic Networks. Wiley, New York.

[18] D.P. Kroese and V.F. Nicola (2002). Efficient simulation of a tandem Jackson network. ACM Transactions of Modeling and Computer Simulation 12 (2) 119141.

[19] V.F. Nicola, P. Shahabuddin, P. Heidelberger and P.W. Glynn (1993). Fast simulation of steady-state availability in non-Markovian highly dependable systems. Proceedings of the Twenty-Third International Symposium on Fault-Tolerant Computing, IEEE Computer Society Press, 38-47.

[20] S. Parekh and J. Walrand (1989). A quick simulation method for excessive backlogs in networks of queues. IEEE Transactions on Automatic Control 34 54-66.

[21] R.Y. Rubinstein (2002). The cross-entropy method and rare events for maximal cut and bipartition problems. ACM Transactions of Modeling and Computer Simulation 12 (1) 27-53.

[22] J.S. Sadowsky (1991). Large deviations theory and efficient simulation of excessive backlogs in a GI/GI/m queue. IEEE Trans. on Automatic Control 36 1383-1394.

[23] M. Villen-Altamirano and J. Villen-Altamirano (2002). Analysis of RESTART simulation: theoretical basis and Sensitivity study. European Transactions on Telecommunications 13 (4) 373-386.

[24] R.R. Weber (1979). The interchangeability

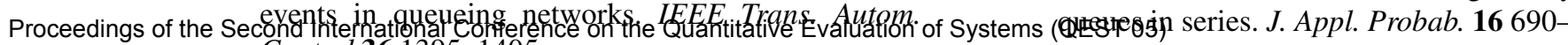

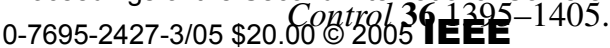




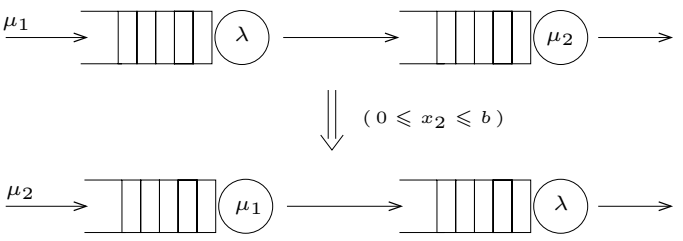

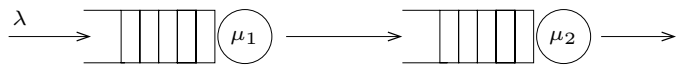

a) Forward time process

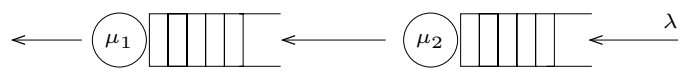

b) Reverse time process

Figure 1. Time reversal of the 2-node tandem network

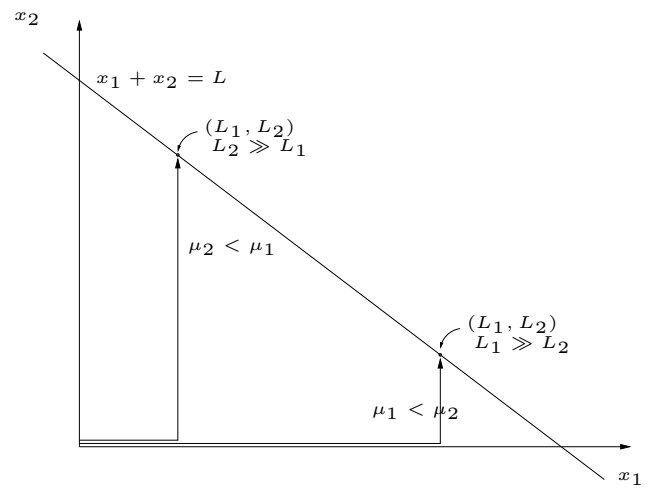

Figure 2. Most likely path to population overflow
Figure 3. SDH change of measure

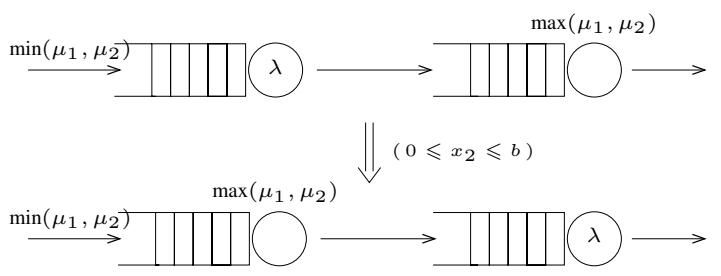

Figure 4. SDH-A change of measure

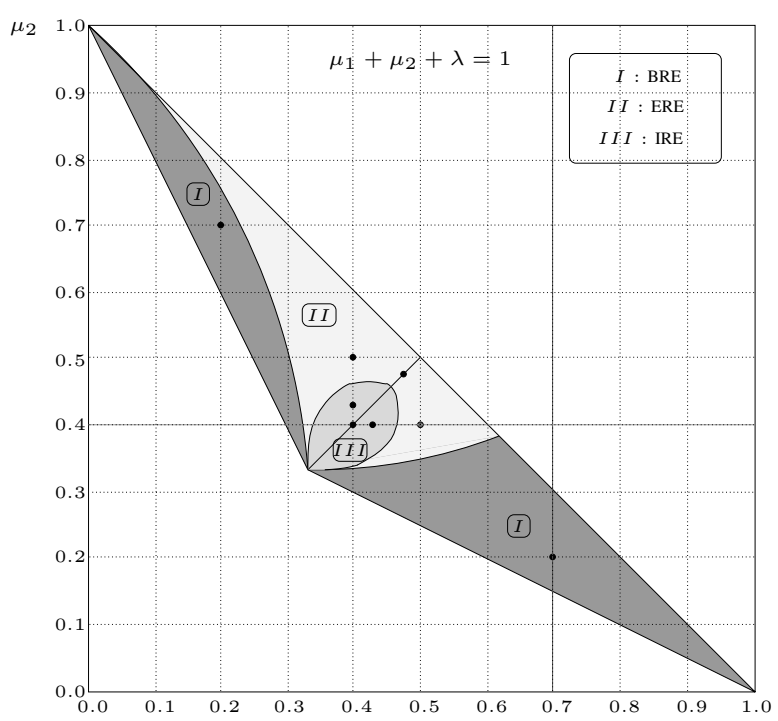

Figure 5. Asymptotic efficiency of PW in the feasible parameter space (as empirically determined in [5]) 
Table 1. BRE Point I-1 $(0.1,0.7,0.2)$

\begin{tabular}{|c|c|c|c|c|c|c|c|}
\hline \multirow[t]{2}{*}{$\overline{\mathbf{L}}$} & \multirow{2}{*}{$\begin{array}{c}\text { Numerical } \\
\gamma(L)\end{array}$} & PW & \multicolumn{2}{|r|}{ SDA } & \multicolumn{3}{|c|}{ SDH $\equiv$ SDH-A } \\
\hline & & $\tilde{\gamma}(L) \pm R E \%$ & b & $\tilde{\gamma}(L) \pm R E \%$ & b & $\tilde{\gamma}(L) \pm R E \%$ & $V R R$ \\
\hline 25 & $4.1723-08$ & $4.1813 \mathrm{e}-08 \pm 0.09$ & 6 & $4.1724 \mathrm{e}-08 \pm 0.005$ & 0 & $4.1772 \mathrm{e}-008 \pm 0.05$ & 0.19 \\
\hline 50 & $1.2435-15$ & $1.2438 \mathrm{e}-15 \pm 0.09$ & 6 & $1.2434 \mathrm{e}-15 \pm 0.003$ & 0 & $1.2435 \mathrm{e}-015 \pm 0.05$ & 0.04 \\
\hline 100 & $1.1044-30$ & $1.1057 \mathrm{e}-30 \pm 0.09$ & 6 & $1.1044 \mathrm{e}-30 \pm 0.004$ & 0 & $1.1042 \mathrm{e}-030 \pm 0.05$ & 0.03 \\
\hline
\end{tabular}

Table 2. BRE Point I-2 $(0.1,0.2,0.7)$

\begin{tabular}{|c|c|c|c|c|c|c|c|c|c|c|}
\hline \multirow[t]{2}{*}{$\overline{\mathbf{L}}$} & \multirow{2}{*}{$\begin{array}{c}\text { Numerical } \\
\gamma(L)\end{array}$} & PW & \multicolumn{2}{|r|}{ SDA } & \multicolumn{3}{|c|}{ SDH } & \multicolumn{3}{|c|}{ SDH-A } \\
\hline & & $\tilde{\gamma}(L) \pm R E \%$ & $\mathbf{b}$ & $\tilde{\gamma}(L) \pm R E \%$ & $\mathbf{b}$ & $\tilde{\gamma}(L) \pm R E \%$ & $V R R$ & $\mathbf{b}$ & $\tilde{\gamma}(L) \pm R E \%$ & $V R R$ \\
\hline 25 & $4.1723 \mathrm{e}-08$ & $4.1614 \mathrm{e}-08 \pm 0.12$ & 3 & $4.1703 \mathrm{e}-08 \pm 0.06$ & $\infty$ & $4.1713 \mathrm{e}-08 \pm 0.10$ & 2.68 & $\infty$ & $4.1797 \mathrm{e}-08 \pm 0.11$ & 1.93 \\
\hline 50 & $1.2435 \mathrm{e}-15$ & $1.2445 \mathrm{e}-15 \pm 0.12$ & 3 & $1.2172 \mathrm{e}-15 \pm 0.06$ & $\infty$ & $1.2419 \mathrm{e}-15 \pm 0.10$ & 1.61 & $\infty$ & $1.2432 \mathrm{e}-15 \pm 0.11$ & 1.30 \\
\hline 100 & $1.1044 \mathrm{e}-30$ & $1.1038 \mathrm{e}-30 \pm 0.12$ & 3 & $1.0828 \mathrm{e}-30 \pm 0.06$ & $\infty$ & $1.1043 \mathrm{e}-30 \pm 0.10$ & 1.36 & $\infty$ & $1.1054 \mathrm{e}-30 \pm 0.11$ & 1.13 \\
\hline
\end{tabular}

Table 3. ERE Point II-1 $(0.1,0.5,0.4)$

\begin{tabular}{|c|c|c|c|c|c|c|c|c|c|c|}
\hline \multirow[t]{2}{*}{$\mathbf{L}$} & \multirow{2}{*}{$\begin{array}{c}\text { Numerical } \\
\gamma(L)\end{array}$} & $\overline{\mathbf{P W} *}$ & \multicolumn{2}{|r|}{ SDA } & \multicolumn{3}{|c|}{ SDH } & \multicolumn{3}{|c|}{ SDH-A } \\
\hline & & $\tilde{\gamma}(L) \pm R E \%$ & b & $\tilde{\gamma}(L) \pm R E \%$ & $\mathbf{b}$ & $\tilde{\gamma}(L) \pm R E \%$ & $V R R$ & b & $\tilde{\gamma}(L) \pm R E \%$ & $\overline{V R R}$ \\
\hline 25 & $1.3269 \mathrm{e}-14$ & $1.3897 \mathrm{e}-14 \pm 5.02$ & 5 & $1.3270 \mathrm{e}-14 \pm 0.006$ & 3 & $1.3251 \mathrm{e}-14 \pm 0.13$ & 0.04 & 2 & $1.3267 \mathrm{e}-14 \pm 0.10$ & 0.07 \\
\hline 50 & $1.1833 \mathrm{e}-29$ & $1.1645 \mathrm{e}-29 \pm 0.75$ & 5 & $1.1833 \mathrm{e}-29 \pm 0.003$ & 3 & $1.1822 \mathrm{e}-29 \pm 0.13$ & 0.01 & 2 & $1.1842 \mathrm{e}-29 \pm 0.09$ & 0.01 \\
\hline 100 & $9.3345 e-60$ & $9.2549 \mathrm{e}-60 \pm 0.97$ & 5 & $9.3344 \mathrm{e}-60 \pm 0.006$ & 3 & $9.3438 \mathrm{e}-60 \pm 0.13$ & 0.02 & 2 & $9.3183 \mathrm{e}-60 \pm 0.09$ & 0.03 \\
\hline
\end{tabular}

Table 4. ERE Point II-2 $(0.1,0.4,0.5)$

\begin{tabular}{|c|c|c|c|c|c|c|c|c|c|c|}
\hline \multirow[t]{2}{*}{$\mathbf{L}$} & \multirow{2}{*}{$\begin{array}{c}\text { Numerical } \\
\gamma(L)\end{array}$} & $\overline{\mathbf{P W} *}$ & \multicolumn{2}{|r|}{ SDA } & \multicolumn{3}{|c|}{ SDH } & \multicolumn{3}{|c|}{ SDH-A } \\
\hline & & $\tilde{\gamma}(L) \pm R E \%$ & b & $\tilde{\gamma}(L) \pm R E \%$ & b & $\tilde{\gamma}(L) \pm R E \%$ & $V R R$ & b & $\tilde{\gamma}(L) \pm R E \%$ & $\overline{V R R}$ \\
\hline 25 & $1.3269 \mathrm{e}-14$ & $1.1333 \mathrm{e}-14 \pm 4.95$ & 4 & $1.3305 \mathrm{e}-14 \pm 0.13$ & 7 & $1.3298 \mathrm{e}-14 \pm 0.20$ & 6.86 & 7 & $1.3275 \mathrm{e}-14 \pm 0.20$ & 7.05 \\
\hline 50 & $1.1833 \mathrm{e}-29$ & $1.1576 \mathrm{e}-29 \pm 10.5$ & 4 & $1.1835 \mathrm{e}-29 \pm 0.19$ & 7 & $1.1865 \mathrm{e}-29 \pm 0.26$ & 5.23 & 7 & $1.1794 \mathrm{e}-29 \pm 0.27$ & 4.49 \\
\hline 100 & $9.3345 \mathrm{e}-60$ & $8.8379 \mathrm{e}-60 \pm 11.2$ & 4 & $9.3161 \mathrm{e}-60 \pm 0.34$ & 7 & $9.3032 \mathrm{e}-60 \pm 0.40$ & 4.12 & 7 & $9.2646 \mathrm{e}-60 \pm 0.48$ & 2.58 \\
\hline
\end{tabular}

Table 5. IRE Point III-1 $(0.18,0.42,0.4)$

\begin{tabular}{|c|c|c|c|c|c|c|c|c|c|c|}
\hline \multirow[t]{2}{*}{$\mathbf{L}$} & \multirow{2}{*}{$\begin{array}{c}\text { Numerical } \\
\gamma(L)\end{array}$} & $\mathbf{P W} *$ & \multicolumn{2}{|r|}{ SDA } & \multicolumn{3}{|c|}{ SDH } & \multicolumn{3}{|c|}{ SDH-A } \\
\hline & & $\tilde{\gamma}(L) \pm R E \%$ & b & $\tilde{\gamma}(L) \pm R E \%$ & b & $\tilde{\gamma}(L) \pm R E \%$ & $\nabla R R$ & b & $\tilde{\gamma}(L) \pm R E \%$ & $\nabla R R$ \\
\hline 25 & $3.8066 \mathrm{e}-08$ & $4.2325 \mathrm{e}-08 \pm 8.53$ & 7 & $3.8066 \mathrm{e}-08 \pm 0.03$ & 6 & $3.8107 \mathrm{e}-08 \pm 0.10$ & 2.22 & 5 & $3.8048 \mathrm{e}-08 \pm 0.08$ & 3.50 \\
\hline 50 & $1.0684 \mathrm{e}-16$ & $1.1551 \mathrm{e}-16 \pm 13.8$ & 7 & $1.0687 \mathrm{e}-16 \pm 0.03$ & 6 & $1.0695 \mathrm{e}-16 \pm 0.08$ & 1.33 & 5 & $1.0693 \mathrm{e}-16 \pm 0.07$ & 1.91 \\
\hline 100 & $5.3355 \mathrm{e}-34$ & $3.8945 \mathrm{e}-34 \pm 3.00$ & 7 & $5.3361 \mathrm{e}-34 \pm 0.03$ & 6 & $5.3397 \mathrm{e}-34 \pm 0.07$ & 0.17 & 5 & $5.3374 \mathrm{e}-34 \pm 0.07$ & 0.20 \\
\hline
\end{tabular}

Table 6. IRE Point III-2 $(0.18,0.4,0.42)$

\begin{tabular}{|c|c|c|c|c|c|c|c|c|c|c|}
\hline \multirow[t]{2}{*}{$\mathbf{L}$} & \multirow{2}{*}{$\begin{array}{c}\text { Numerical } \\
\gamma(L)\end{array}$} & $\mathbf{P W} *$ & \multicolumn{2}{|r|}{ SDA } & \multicolumn{3}{|c|}{ SDH } & \multicolumn{3}{|c|}{ SDH-A } \\
\hline & & $\tilde{\gamma}(L) \pm R E \%$ & b & $\tilde{\gamma}(L) \pm R E \%$ & b & $\tilde{\gamma}(L) \pm R E \%$ & $V R R$ & b & $\tilde{\gamma}(L) \pm R E \%$ & $V R R$ \\
\hline 25 & $3.8066 \mathrm{e}-08$ & $3.1401 \mathrm{e}-08 \pm 6.77$ & 5 & $3.8056 \mathrm{e}-08 \pm 0.08$ & 9 & $3.8080 \mathrm{e}-08 \pm 0.13$ & 5.05 & 9 & $3.8023 \mathrm{e}-08 \pm 0.13$ & 5.61 \\
\hline 50 & $1.0684 \mathrm{e}-16$ & $5.7496 \mathrm{e}-17 \pm 3.54$ & 5 & $1.0693 \mathrm{e}-16 \pm 0.12$ & 9 & $1.0699 \mathrm{e}-16 \pm 0.14$ & 5.40 & 9 & $1.0664 \mathrm{e}-16 \pm 0.14$ & 5.49 \\
\hline 100 & $5.3355 \mathrm{e}-34$ & $2.9055 \mathrm{e}-34 \pm 4.52$ & 5 & $5.3363 \mathrm{e}-34 \pm 0.26$ & 9 & $5.3338 \mathrm{e}-34 \pm 0.26$ & 4.58 & 9 & $5.3215 \mathrm{e}-34 \pm 0.27$ & 3.98 \\
\hline
\end{tabular}

Table 7. ERE Point IV-1 $(0.04,0.48,0.48)$

\begin{tabular}{||c||c||c||c|c||c|c|c||}
\hline \multicolumn{1}{||c||}{$\mathbf{L}$} & Numerical & PW $*$ & \multicolumn{2}{|c||}{ SDA } & \multicolumn{3}{|c||}{ SDH $\equiv$ SDH-A } \\
\cline { 3 - 9 } & $\gamma(L)$ & $\tilde{\gamma}(L) \pm R E \%$ & $\mathbf{b}$ & $\tilde{\gamma}(L) \pm R E \%$ & $\mathbf{b}$ & $\tilde{\gamma}(L) \pm R E \%$ & $V R R$ \\
\hline 25 & $2.8722 \mathrm{e}-025$ & $2.6050 \mathrm{e}-025 \pm 8.64$ & 3 & $2.8711 \mathrm{e}-025 \pm 0.06$ & 3 & $2.8721 \mathrm{e}-025 \pm 0.13$ & 3.29 \\
\hline 50 & $6.0327 \mathrm{e}-052$ & $2.3672 \mathrm{e}-052 \pm 4.67$ & 3 & $6.0248 \mathrm{e}-052 \pm 0.08$ & 3 & $6.0399 \mathrm{e}-052 \pm 0.12$ & 4.65 \\
\hline 100 & $1.3270 \mathrm{e}-105$ & $3.5984 \mathrm{e}-106 \pm 19.5$ & 3 & $1.3274 \mathrm{e}-105 \pm 0.08$ & 3 & $1.3288 \mathrm{e}-105 \pm 0.16$ & 1.19 \\
\hline
\end{tabular}

Table 8. IRE Point IV-2 $(0.2,0.4,0.4)$

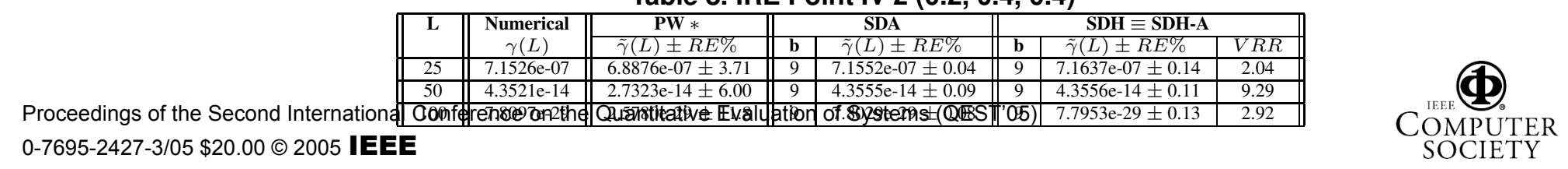

